\title{
Structure of the time projection for stopping times in von Neumann algebras
}

\author{
Andrzej Łuczak* \\ Faculty of Mathematics \\ Łódź University \\ ul. Banacha 22 \\ 90-238 Łodź, Poland \\ anluczak@math.uni.lodz.pl
}

\begin{abstract}
We give an explicit formula for the time projection in an arbitrary von Neumann algebra from which all its basic properties can be easily derived. The analysis of the situation when this time projection is a conditional expectation is also performed.
\end{abstract}

\section{Introduction}

The aim of these notes is to investigate some properties of the time projection for a stopping time in a von Neumann algebra. This is done solely by using an explicit formula for the projection, without any reference to stochastic integration. In particular, we obtain simple conditions for stopping a noncommutative martingale. The problem of when the time projection can be treated as a conditional expectation is also addressed. Its solution, known in the case of the Clifford probability gauge space, is thus generalised to a fairly general context.

\section{Preliminaries and notation}

Throughout the paper $\mathcal{A}$ will denote a von Neumann algebra acting in a Hilbert space $\mathcal{H}$ with a cyclic and separating vector $\Omega$. $\omega$ will stand for a (normal faithful) vector state on $\mathcal{A}$ induced by $\Omega$. Let $\left(\mathcal{A}_{t}: t \geq 0\right)$ be a filtration of $\mathcal{A}$, i.e. an increasing net of von Neumann subalgebras of $\mathcal{A}$ such that $\mathcal{A}=\mathcal{A}_{\infty}:=$ $\left(\cup_{t \geq 0} \mathcal{A}_{t}\right)^{\prime \prime}$. We assume that there are normal conditional expectations $\mathbb{E}_{t}, t \geq 0$, from $\mathcal{A}$ onto $\mathcal{A}_{t}$ leaving $\omega$ invariant. It follows easily (cf.[2, Proposition 1.2]) that if we define

$$
P_{t}(x \Omega)=\left(\mathbb{E}_{t} x\right) \Omega, x \in \mathcal{A},
$$

*Work supported by KBN grant 2 PO3A 04410 
then $P_{t}$ is a projection from $\mathcal{H}$ onto $\mathcal{H}_{t}=\overline{\mathcal{A}_{t} \Omega}$, consequently, $P_{t} \in \mathcal{A}_{t}^{\prime}$; we have also $\mathbb{E}_{t} \mathbb{E}_{s}=\mathbb{E}_{s} \mathbb{E}_{t}=\mathbb{E}_{s \wedge t}$. In what follows we shall be concerned with the "time parameter" $t$ belonging either to the interval $[0,+\infty)$ or to the interval $[0, u]$, where $0<u \leq+\infty$. Accordingly, we adopt the following definition. A (quantum, noncommutative) stopping time $\tau$ is an increasing net $\left(q_{t}\right), t \in$ $[0,+\infty)$ or $[0,+\infty]$ of projections such that $q_{t} \in \mathcal{A}_{t}, q_{0}=0$, and $\bigvee_{t>0} q_{t}=\mathbf{1}$ in the case $t \in[0,+\infty)$ or $q_{\infty}=\mathbf{1}$ in the case $t \in[0,+\infty]$. The definition above is a proper generalisation of the notion of the classical (commutative) stopping time (cf. 11, 2, 3, - 由 for more information). A fairly general theory of stopping a noncommutative process has so far been achieved only for martingales. Let us briefly recall its main points here.

A martingale in $\mathcal{H}$ is a process $(\xi(t): t \geq 0)$ such that $\xi(t) \in \mathcal{H}_{t}$ and for each $s, t \geq 0, s \leq t$,

$$
P_{s} \xi(t)=\xi(s) .
$$

If we allow $t \in[0,+\infty]$ then it follows that there is $\xi(=\xi(\infty))$ such that $\xi(t)=$ $P_{t} \xi$; such martingales are called closed, and it is not difficult to see that the following conditions are equivalent: (i) $(\xi(t))$ is closed (ii) $\sup _{t}\|\xi(t)\|<+\infty$ (iii) there exists $\lim _{t \rightarrow \infty} \xi(t)$ (cf. 目, Proposition 1.1]).

Now stopping $(\xi(t))$ consists in the following procedure. For interval $[0, u](u=$ $+\infty$ if $(\xi(t))$ is closed) we consider its partition $\theta=\left\{0=t_{0}<t_{1}<\cdots<t_{n}=\right.$ $u\}$, and form the sum

$$
\xi_{\tau(\theta)}=\sum_{i=1}^{n}\left(q_{t_{i}}-q_{t_{i-1}}\right) \xi\left(t_{i}\right) .
$$

Taking the limit of the net $\left\{\xi_{\tau(\theta)}: \theta\right.$ - partition $\}$ as $\theta$ refines, gives us the stopped element $\xi_{\tau}(u)$, which is all we need if $u=+\infty$; however, if $u<+\infty$ it seems reasonable to define $\xi_{\tau}$ as $\lim _{u \rightarrow \infty} \xi_{\tau}(u)$.

The existence of the two limits above is by no means obvious. It turns out that while the limit in (11) does exist it need not be so with the other one, and thus we are guaranteed only of the possibility of stopping a closed martingale. To analyse $\xi_{\tau(\theta)}$ observe that the martingale property yields

$$
\xi\left(t_{i}\right)=P_{t_{i}} \xi(u), i=1, \ldots, n,
$$

and hence

$$
\xi_{\tau(\theta)}=\sum_{i=1}^{n}\left(q_{t_{i}}-q_{t_{i-1}}\right) P_{t_{i}} \xi(u) .
$$

Put

$$
M_{\tau(\theta)}(u)=\sum_{i=1}^{n}\left(q_{t_{i}}-q_{t_{i-1}}\right) P_{t_{i}} .
$$

Then $M_{\tau(\theta)}(u)$ is a projection in $\mathcal{H}$ (recall that $\left.P_{t_{i}} \in \mathcal{A}_{t_{i}}^{\prime}, q_{t_{i-1}}, q_{t_{i}} \in \mathcal{A}_{t_{i}}\right)$. It is easily seen that the net $\left\{M_{\tau(\theta)}(u): \theta\right.$ - partition $\}$ decreases, so there exists 
$\lim _{\theta} M_{\tau(\theta)}(u)$ which we denote by $M_{\tau}(u)$ and call the time projection; it is also clear that

$$
M_{\tau}(u)=\bigwedge_{\theta} M_{\tau(\theta)}(u) .
$$

Accordingly, we have by (2)

$$
\xi_{\tau}(u)=\lim _{\theta} \xi_{\tau(\theta)}=\lim _{\theta} M_{\tau(\theta)}(u) \xi(u)=M_{\tau}(u) \xi(u) .
$$

If $u=+\infty$ we shall write $M_{\tau}$ instead of $M_{\tau}(\infty)$; note that this is the case considered in [1, 3, 团] and mainly in [2]. However, in [2] a more general setting that we have defined above is also taken into account.

As a final remark let us observe that the definition of the time projection as well as the results of the next section could be obtained for Haagerup's $L^{2}(\mathcal{A}, \omega)$-space and the algebra $\mathcal{A}$ acting on it by left multiplication, especially in view of a spatial isomorphism between the representations $(\mathcal{A}, \mathcal{H}, \Omega)$ and $\left(\mathcal{A}, L^{2}(\mathcal{A}, \omega), h_{\omega}^{1 / 2}\right)$ where $h_{\omega}^{1 / 2}$ is a cyclic and separating vector in $L^{2}(\mathcal{A}, \omega)$. The reasons for which we have adopted a more traditional approach lie in Section 3. There we want to treat the time projection, which is a projection in a Hilbert space, as a projection in the algebra $\mathcal{A}$, and passing from one to another is much more straightforward in our original setup where we have a natural embedding of $\mathcal{A}$ into $\mathcal{H}$ given by $\mathcal{A} \ni x \mapsto x \Omega \in \mathcal{H}$.

\section{Representation of the time projection}

In this section we analyse various properties of the time projection by means of an explicit formula expressing it in terms of the $P_{t}$ and $q_{t}$.

Theorem 1 Let $u \in(0,+\infty]$. Then

$$
M_{\tau}(u)=\bigwedge_{t \leq u}\left(q_{u}-q_{t} P_{t}^{\perp}\right) .
$$

Proof. Take the partition $\theta_{0}=\left\{0=t_{0}<t_{1}=u\right\}$. We have

$$
M_{\tau}(u) \leq M_{\tau\left(\theta_{0}\right)}=\left(q_{t_{1}}-q_{t_{0}}\right) P_{t_{1}}=q_{u} P_{u} \leq q_{u} .
$$

Let $\xi \in \mathcal{H}$, and assume that $M_{\tau}(u) \xi=q_{u} \xi$. For an arbitrary $t \in[0, u]$ we have

$$
M_{\tau}(u) \leq\left(q_{t}-q_{0}\right) P_{t}+\left(q_{u}-q_{t}\right) P_{u} \leq q_{u},
$$

giving the equality

$$
\left(q_{t}-q_{0}\right) P_{t} \xi+\left(q_{u}-q_{t}\right) P_{u} \xi=q_{u} \xi .
$$

Applying $q_{t}$ to both sides yields

$$
q_{t} P_{t} \xi=q_{t} \xi
$$


Conversely, if for each $t \in[0, u]$ equality (雨) holds, then for any $s \leq t$ we have, applying $q_{s}$ to both sides of (4),

$$
q_{s} P_{t} \xi=q_{s} \xi
$$

and for any partition $\theta=\left\{0=t_{0}<t_{1}<\cdots<t_{n}=u\right\}$

$$
M_{\tau(\theta)}(u) \xi=\sum_{i=1}^{n}\left(q_{t_{i}}-q_{t_{i-1}}\right) P_{t_{i}} \xi=\sum_{i=1}^{n}\left(q_{t_{i}} \xi-q_{t_{i-1}} \xi\right)=q_{t_{n}} \xi-q_{t_{0}} \xi=q_{u} \xi
$$

hence

$$
M_{\tau}(u) \xi=\lim _{\theta} M_{\tau(\theta)}(u) \xi=q_{u} \xi .
$$

We have thus obtained equivalence of the following conditions:

(i) $M_{\tau}(u) \xi=q_{u} \xi$

(ii) for each $t \in[0, u] \quad q_{t} P_{t} \xi=q_{t} \xi$,

or put in another way

(i') $\left[q_{u}-M_{\tau}(u)\right] \xi=0$

(ii') for each $t \in[0, u] \quad q_{t} P_{t}^{\perp} \xi=0$.

But condition (ii') is equivalent to the equality

$$
\left(\bigvee_{t \leq u} q_{t} P_{t}^{\perp}\right) \xi=0
$$

which means that the projections $q_{u}-M_{\tau}(u)$ and $\bigvee_{t \leq u} q_{t} P_{t}^{\perp}$ have the same null spaces, so they must be equal:

$$
q_{u}-M_{\tau}(u)=\bigvee_{t \leq u} q_{t} P_{t}^{\perp} .
$$

Consequently,

$$
M_{\tau}(u)=q_{u}-\bigvee_{t \leq u} q_{t} P_{t}^{\perp}=\bigwedge_{t \leq u}\left(q_{u}-q_{t} P_{t}^{\perp}\right) .
$$

Corollary 1 If $u=+\infty$ then

$$
M_{\tau}=\bigwedge_{t \geq 0}\left(q_{t}^{\perp}+q_{t} P_{t}\right)
$$


Indeed, we then have

$$
q_{\infty}-q_{t} P_{t}^{\perp}=\mathbf{1}-q_{t} P_{t}^{\perp}=q_{t}^{\perp}+q_{t} P_{t},
$$

and for $t=+\infty$

$$
q_{\infty}^{\perp}+q_{\infty} P_{\infty}=\mathbf{1}
$$

giving

$$
M_{\tau}=M_{\tau}(\infty)=\bigwedge_{0 \leq t \leq+\infty}\left(q_{\infty}-q_{t} P_{t}^{\perp}\right)=\bigwedge_{0 \leq t \leq+\infty}\left(q_{t}^{\perp}+q_{t} P_{t}\right)=\bigwedge_{0 \leq t<+\infty}\left(q_{t}^{\perp}+q_{t} P_{t}\right) .
$$

Theorem 2 Let the set $\left\{M_{\tau} \xi(t): t \in[0,+\infty)\right\}$ be norm-bounded. Then the martingale $(\xi(t))$ can be stopped and

$$
\xi_{\tau}=\lim _{t \rightarrow \infty} M_{\tau} \xi(t)
$$

Proof. Put

$$
\eta(t)=M_{\tau} \xi(t) .
$$

For each $s, t \in[0,+\infty)$ we have

$$
P_{s}\left(q_{t}^{\perp}+q_{t} P_{t}\right)=\left\{\begin{array}{ll}
P_{s} q_{t}^{\perp}+P_{s} q_{t}=P_{s} & \text { for } s \leq t \\
P_{s} q_{t}^{\perp}+q_{t} P_{t} & \text { for } s>t
\end{array}=\left(q_{t}^{\perp}+q_{t} P_{t}\right) P_{s},\right.
$$

and from (5) we get

$$
P_{s} M_{\tau}=M_{\tau} P_{s}
$$

If $s \leq t$, then

$$
P_{s} \eta(t)=P_{s} M_{\tau} \eta(t)=M_{\tau} P_{s} \eta(s)=M_{\tau} \eta(s)=\eta(s),
$$

which shows that $(\eta(t))$ is a martingale, and since it is norm-bounded, we have $\eta(t) \rightarrow \eta$, as $t \rightarrow \infty$, for some $\eta \in \mathcal{H}$. From (6) we have

$$
M_{\tau}(t) \eta(t)=M_{\tau}(t) M_{\tau} \xi(t)=M_{\tau}(t) \xi(t)=\xi_{\tau}(t) .
$$

Now

$$
\begin{aligned}
& \left\|M_{\tau}(t) \eta(t)-M_{\tau} \eta\right\| \leq\left\|M_{\tau}(t)[\eta(t)-\eta]\right\|+\left\|\left[M_{\tau}(t)-M_{\tau}\right] \eta\right\| \leq \\
& \quad \leq\|\eta(t)-\eta\|+\left\|\left[M_{\tau}(t)-M_{\tau}\right] \eta\right\| \rightarrow 0,
\end{aligned}
$$

since $\lim _{t \rightarrow \infty} M_{\tau}(t)=M_{\tau}$, consequently

$$
\xi_{\tau}(t)=M_{\tau}(t) \eta(t) \rightarrow M_{\tau} \eta .
$$

But $M_{\tau} \eta(t)=\eta(t)$, and thus $M_{\tau} \eta=\eta$, giving

$$
\xi_{\tau}=\lim _{t \rightarrow \infty} \xi_{\tau}(t)=M_{\tau} \eta=\eta=\lim _{t \rightarrow \infty} M_{\tau} \xi(t) .
$$

Observe that the result of the last theorem perfectly agrees with what we have for a closed martingale where also

$$
\xi_{\tau}=M_{\tau} \xi=\lim _{t \rightarrow \infty} M_{\tau} \xi(t) .
$$




\section{Time projection as a conditional expectation}

In this section we consider a question when the time projection can be treated as a conditional expectation. A problem of this type was analysed in 1] for the Clifford probability gauge space and solved by using some properties of the Clifford quantum stochastic integral. The solution we give here works in the general context of an arbitrary von Neumann algebra; moreover it is simple and does not employ any theory of stochastic integration.

Let $\tau=\left(q_{t}: t \in[0,+\infty]\right)$ be a stopping time, and let $M_{\tau}$ be the time projection. $M_{\tau}$ can be treated as a conditional expectation if

$$
M_{\tau}(x \Omega)=y \Omega,
$$

and the map $\mathbb{E}_{\tau}: x \mapsto y$ is a conditional expectation. We then have

$$
\left(\mathbb{E}_{\tau} x\right) \Omega=M_{\tau}(x \Omega) .
$$

Put

$$
\mathcal{B}_{\tau}=\left\{x \in \mathcal{A}: \text { for each } t \geq 0 \quad x q_{t}=q_{t} x\right\}=\mathcal{A} \cap\left\{q_{t}: t \in[0,+\infty]\right\}^{\prime} .
$$

For any partition $\theta=\left\{0=t_{0}<t_{1}<\cdots<t_{n}=+\infty\right\}$ let

$$
\mathcal{A}_{\tau(\theta)}=\left\{x \in \mathcal{A}: x q_{t_{i}}=q_{t_{i}} x \in \mathcal{A}_{t_{i}}, i=0,1, \ldots, n\right\},
$$

and let

$$
\mathcal{A}_{\tau}=\bigcap_{\theta} \mathcal{A}_{\tau(\theta)}=\left\{x \in \mathcal{A}: \text { for each } t \geq 0 x q_{t}=q_{t} x \in \mathcal{A}_{t}\right\} .
$$

Theorem $3 M_{\tau} \mid \mathcal{B}$ is a normal faithful conditional expectation onto $\mathcal{A}_{\tau}$ leaving $\omega$ invariant.

Proof. For a partition $\theta=\left\{0=t_{0}<t_{1}<\cdots<t_{n}=+\infty\right\}$ define on $\mathcal{B}_{\tau}$ the $\operatorname{map} \mathbb{E}_{\tau_{(\theta)}}$ by

$$
\begin{aligned}
& \mathbb{E}_{\tau_{(\theta)}} x=\sum_{i=1}^{n}\left(q_{t_{i}}-q_{t_{i-1}}\right) \mathbb{E}_{t_{i}} x=\sum_{i=1}^{n} \mathbb{E}_{t_{i}}\left(\left(q_{t_{i}}-q_{t_{i-1}}\right) x\right)= \\
& =\sum_{i=1}^{n}\left(\mathbb{E}_{t_{i}} x\right)\left(q_{t_{i}}-q_{t_{i-1}}\right), x \in \mathcal{B}_{\tau} .
\end{aligned}
$$

For each $t \in[0,+\infty]$ we have $t_{j-i} \leq t<t_{j}$ with some $j$, so

$$
\begin{aligned}
& q_{t} \mathbb{E}_{\tau(\theta)} x=q_{t} \sum_{i=1}^{j-1}\left(q_{t_{i}}-q_{t_{i-1}}\right) \mathbb{E}_{t_{i}} x+q_{t}\left(q_{t_{j}}-q_{t_{j-1}}\right) \mathbb{E}_{t_{j}} x+ \\
& +q_{t} \sum_{i=j+1}^{n}\left(q_{t_{i}}-q_{t_{i-1}}\right) \mathbb{E}_{t_{i}} x=\sum_{i=1}^{j-1}\left(q_{t_{i}}-q_{t_{i-1}}\right) \mathbb{E}_{t_{i}} x+\left(q_{t}-q_{t_{j-1}}\right) \mathbb{E}_{t_{j}} x
\end{aligned}
$$


and

$$
\begin{aligned}
& \left(\mathbb{E}_{\tau(\theta)} x\right) q_{t}=\sum_{i=1}^{j-1}\left(\mathbb{E}_{t_{i}} x\right)\left(q_{t_{i}}-q_{t_{i-1}}\right) q_{t}+\left(\mathbb{E}_{t_{j}} x\right)\left(q_{t_{j}}-q_{t_{j-1}}\right) q_{t}+ \\
& +\sum_{i=j+1}^{n}\left(\mathbb{E}_{t_{i}} x\right)\left(q_{t_{i}}-q_{t_{i-1}}\right) q_{t}=\sum_{i=1}^{j-1}\left(\mathbb{E}_{t_{i}} x\right)\left(q_{t_{i}}-q_{t_{i-1}}\right)+\left(\mathbb{E}_{t_{j}} x\right)\left(q_{t}-q_{t_{j-1}}\right) .
\end{aligned}
$$

But for $x \in \mathcal{B}_{\tau}$

$$
\left(q_{t_{i}}-q_{t_{i-1}}\right) \mathbb{E}_{t_{i}} x=\mathbb{E}_{t_{i}}\left(\left(q_{t_{i}}-q_{t_{i-1}}\right) x\right)=\left(\mathbb{E}_{t_{i}} x\right)\left(q_{t_{i}}-q_{t_{i-1}}\right),
$$

and

$$
\left(q_{t}-q_{t_{j-1}}\right) \mathbb{E}_{t_{j}} x=\mathbb{E}_{t_{j}} x\left(\left(q_{t}-q_{t_{j-1}}\right) x\right)=\left(\mathbb{E}_{t_{j}} x\right)\left(q_{t}-q_{t_{j-1}}\right),
$$

which shows that

$$
q_{t} \mathbb{E}_{\tau(\theta)} x=\left(\mathbb{E}_{\tau(\theta)} x\right) q_{t},
$$

i.e. $\mathbb{E}_{\tau(\theta)} x \in \mathcal{B}_{\tau}$. Furthermore, for each $j=0,1, \ldots, n$

$$
q_{t_{j}} \mathbb{E}_{\tau(\theta)} x=\sum_{i=1}^{j}\left(q_{t_{i}}-q_{t_{i-1}}\right) \mathbb{E}_{t_{i}} x=\sum_{i=1}^{j}\left(\mathbb{E}_{t_{i}} x\right)\left(q_{t_{i}}-q_{t_{i-1}}\right)=\left(\mathbb{E}_{\tau(\theta)} x\right) q_{t_{j}},
$$

showing that $\mathbb{E}_{\tau(\theta)} x \in \mathcal{A}_{\tau(\theta)}$. For $x \in \mathcal{A}_{\tau(\theta)}$ we have $\mathbb{E}_{t_{i}} x=x$, hence

$$
\mathbb{E}_{\tau(\theta)} x+\sum_{i=1}^{n}\left(q_{t_{i}}-q_{t_{i-1}}\right) x=x
$$

which means that $\mathbb{E}_{\tau(\theta)}$ is a projection from $\mathcal{B}_{\tau}$ onto $\mathcal{B}_{\tau} \cap \mathcal{A}_{\tau_{(\theta)}}$. If $x \in \mathcal{B}_{\tau}^{+}$, then $\left(q_{t_{i}}-q_{t_{i-1}}\right) x=\left(q_{t_{i}}-q_{t_{i-1}}\right) x\left(q_{t_{i}}-q_{t_{i-1}}\right) \geq 0$, so

$$
\mathbb{E}_{\tau(\theta)} x=\sum_{i=1}^{n}\left(q_{t_{i}}-q_{t_{i-1}}\right) \mathbb{E}_{t_{i}} x=\sum_{i=1}^{n} \mathbb{E}_{t_{i}}\left(\left(q_{t_{i}}-q_{t_{i-1}}\right) x\right) \geq 0,
$$

thus $\mathbb{E}_{\tau(\theta)}$ is positive. Since $\mathbb{E}_{\tau(\theta)} \mathbf{1}=\mathbf{1}$, we infer that $\left\|\mathbb{E}_{\tau(\theta)}\right\|=1$, and by virtue of [5, Theorem 9.1 p.116], $\mathbb{E}_{\tau(\theta)}$ is a conditional expectation. We have

$$
\left(\mathbb{E}_{\tau(\theta)} x\right) \Omega=M_{\tau_{(\theta)}}(x \Omega), x \in \mathcal{B}_{\tau} .
$$

Put

$$
x_{\theta}=\mathbb{E}_{\tau(\theta)} x .
$$

Then $\left\{x_{\theta}\right\}$ is a bounded net of elements in $\mathcal{A}$, and for each $x^{\prime} \in \mathcal{A}^{\prime}$

$$
x_{\theta}\left(x^{\prime} \Omega\right)=x^{\prime}\left(x_{\theta} \Omega\right)=x^{\prime}\left(\mathbb{E}_{\tau(\theta)} x\right) \Omega=x^{\prime} M_{\tau(\theta)}(x \Omega) \rightarrow x^{\prime} M_{\tau}(x \Omega) .
$$

Thus the net $\left\{x_{\theta}\right\}$ converges on the dense subspace $\mathcal{A}^{\prime} \Omega$ of $\mathcal{H}$, and since $\left\|x_{\theta}\right\| \leq\|x\|$, it follows that $\left\{x_{\theta}\right\}$ converges in the strong operator topology on $\mathcal{A}$, consequently, there is $y \in \mathcal{A}$ such that $x_{\theta} \rightarrow y$ strongly. 
Let

$$
\mathbb{E}_{\tau} x=y=\lim _{\theta} x_{\theta}=\lim _{\theta} \mathbb{E}_{\tau(\theta)} x, x \in \mathcal{B}_{\tau} .
$$

Clearly, $\mathbb{E}_{\tau}$ is a linear positive map on $\mathcal{B}_{\tau}$, such that

$$
\left(\mathbb{E}_{\tau} x\right) \Omega=M_{\tau}(x \Omega), x \in \mathcal{B}_{\tau} .
$$

Since $\mathbb{E}_{\tau} \mathbf{1}=\mathbf{1}$, we have $\left\|\mathbb{E}_{\tau}\right\|=1$. For any partition $\theta$ and $x \in \mathcal{B}_{\tau}$,

$$
\mathbb{E}_{\tau(\theta)}\left(\mathbb{E}_{\tau} x\right) \Omega=M_{\tau(\theta)}\left(\left(\mathbb{E}_{\tau} x\right) \Omega\right)=M_{\tau(\theta)} M_{\tau}(x \Omega)=M_{\tau}(x \Omega)=\left(\mathbb{E}_{\tau} x\right) \Omega,
$$

showing that $\mathbb{E}_{\tau(\theta)} \mathbb{E}_{\tau}=\mathbb{E}_{\tau}$, since $\Omega$ is separating.

Accordingly, $\mathbb{E}_{\tau} x \in \mathcal{A}_{\tau(\theta)}$ for each $\theta$, and it follows that $\mathbb{E}_{\tau} x \in \bigcap_{\theta} \mathcal{A}_{\tau(\theta)}=\mathcal{A}_{\tau}$. Furthermore, if $x \in \mathcal{A}_{\tau}$, then $\mathbb{E}_{\tau(\theta)} x=x$ for each $\theta$, so

$$
\mathbb{E}_{\tau} x=\lim _{\theta} \mathbb{E}_{\tau(\theta)} x=x,
$$

which means that $\mathbb{E}_{\tau}$ is a projection onto $\mathcal{A}_{\tau}$, and thus a conditional expectation. From the equality

$$
\omega \circ \mathbb{E}_{\tau(\theta)}=\omega,
$$

we obtain

$$
\omega \circ \mathbb{E}_{\tau}=\omega,
$$

which, since $\mathbb{E}_{\tau}$ is positive, implies faithfulness and normality of $\mathbb{E}_{\tau}$.

Let us observe that in an entirely analogous way we can obtain a corresponding result for the time projection $M_{\tau}(u)$.

Indeed, putting

$$
\begin{gathered}
\mathcal{B}_{\tau}(u)=\left\{x \in \mathcal{A}: \text { for each } t \leq u \quad x q_{t}=q_{t} x\right\} \\
\mathcal{A}_{\tau}(u)=\left\{x \in \mathcal{A}: \text { for each } t \leq u \quad x q_{t}=q_{t} x \in \mathcal{A}_{t}\right\},
\end{gathered}
$$

we get that $M_{\tau}(u) \mid \mathcal{B}_{\tau}(u)$ is a conditional expectation onto $q_{u} \mathcal{A}_{\tau}(u) q_{u}$.

\section{References}

[1] C. Barnett, T. Lyons, Stopping non-commutative processes, Math. Proc. Cambridge Philos. Soc. 99(1986), 151-161.

[2] C. Barnett, B. Thakrar, Time projections in a von Neumann algebra, J. Operator Theory 10(1987), 19-31.

[3] C. Barnett, B. Thakrar, A non-commutative random stopping theorem, J. Funct. Anal. 88(1990), 342-350.

[4] C. Barnett, I. F. Wilde, Random times and time projections, Proc. Amer. Math. Soc. 110(1990), 425-440.

[5] S. Stratila, Modular Theory in Operator Algebras, Editura Academiei, Bucuresti and Abacus Press, Tunbridge Wells, 1981. 